\title{
ShopRight: A Game-Based Approach for Motivating Healthy Shopping Habits
}

\author{
Ifeoma Adaji \\ University of Saskatchewan \\ Saskatchewan, Canada \\ ifeoma.adaji@usask.ca
}

\author{
Kiemute Oyibo \\ University of Saskatchewan \\ Saskatchewan, Canada \\ Kiemute.oyibo@usask.ca
}

\author{
Julita Vassileva \\ University of Saskatchewan \\ Saskatchewan, Canada \\ jiv@cs.usask.ca
}

\begin{abstract}
Unhealthy eating habits has been identified as one of the major causes of obesity and weight gain in people. When consumers shop for unhealthy foods, they will likely eat unhealthy foods, thus it is imperative to encourage healthy shopping habits among people. To contribute to ongoing research in influencing healthy shopping habits, we present the prototype of ShopRight, a game that encourages healthy shopping habits in consumers. In ShopRight, points are awarded when players "shop" for healthy foods presented to them and "destroy" the unhealthy options as quickly as possible. The aim of the game is to influence shoppers to quickly and easily identify and differentiate between healthy and unhealthy foods when shopping.
\end{abstract}

Healthy shopping, healthy foods, shopping game

\section{INTRODUCTION}

According to Statistics Canada, one in four Canadians are considered to be overweight; this represents over 14 million people in total [1]. This figure has increased by $17.5 \%$ since 2003 and is expected to increase over the next decade ${ }^{1}$. Adopting a non-sedentary lifestyle and embracing healthy eating habits have been identified as the two major ways of improving one's health, reducing weight and preventing weight gain [2]. There has been a lot of research on promoting non-sedentary lifestyles such as the development of games that influence people to be more active [3],[4] and the development of fitness apps and devices such as the Fitbit that encourage people to exercise more and adopt an active lifestyle. However, the area of influencing people to adopt healthier eating habits by shopping healthy is still under-researched. Because people are more likely to eat what they buy, we propose that influencing people to adopt healthier shopping habits (shop for healthier products) could lead them to embrace healthier eating habits.

In 2017, there was a $23 \%$ increase in the number of people who shop online ${ }^{3}$. This growth is projected to continue over the next decade with more companies having an online presence. Retail companies

\footnotetext{
${ }^{1}$ https://www.statcan.gc.ca/tables-tableaux/sum-

som/101/cst01/health81a-eng.htm

${ }^{2}$ www.fitbit.com

${ }^{3}$ https://www.smartinsights.com/digital-marketing-

strategy/online-retail-sales-growth/
}

especially have joined this trend with many retail companies offering same-day delivery to their ecommerce shoppers. This trend has seen an increase in the number of people who shop online for groceries and food products. With more people shopping for groceries online, we propose that implementing the right influence strategies on the ecommerce shopping site could motivate econsumers to shop for healthier foods and thus lead them to eat healthier foods.

One of such influence strategies is the use of games to bring about the desired behaviour change in consumers. Several games such as OrderUp [4] have been developed to promote healthy living but are focused on influencing people to select healthy foods from a menu. In OrderUp, the player takes on the role of a server in a restaurant whose goal is to recommend the healthiest meal from a menu item as quickly as possible. While this strategy could work for people who eat out, it would not be effective when people have to shop for and cook their own foods. Though there has been an increase in how much people spend eating out, families in Canada still spend most of their food budget (about 70\%) cooking at home ${ }^{4}$. Time to Eat [5] is another game that promotes healthy eating habits. In Time to Eat, players are assigned a virtual pet that they have to care for. The players care for their pets by sending pictures of the foods they (the players) eat during the day. The healthier the food, the well cared for the pet

\footnotetext{
${ }^{4}$ https://www.dal.ca/news/2017/12/13/canadians-will-spendmore-in-restaurants-in-2018--canada-s-food-.html
} 
is. While this game can influence healthy eating habits, we believe that tackling the problem at the roots will be more effective; if people are able to shop for healthy foods, they will likely cook healthy foods, thus it is important to influence consumers at the point of shopping to buy healthy foods.

To contribute to research in this area, we present the prototype of ShopRight, a game that promotes healthy shopping habits among e-commerce shoppers. ShopRight allows players to "shop" for food in the game. Players can "improve their health" when they shop for healthy food choices such as fruits and vegetables. They can also "improve their health" when they "destroy" unhealthy foods such as pizza and ice cream. If on the other hand, the player "destroys" healthy foods such as fruits instead of shopping for them, the player's health decreases. In the context of this game, healthy foods are defined as fruits and vegetables. We defined healthy foods this way because the Centre for Disease Control and Prevention recommends a diet with more fruits and vegetables (and less salt, saturated fat, and added sugar) as one of six strategies to live a hearthealthy lifestyle ${ }^{5}$.

We also present in this study the initial response from people who played the game or have seen it being played. Kindly note that this is not the final version of the game but the initial prototype. The game will be modified and improved in the future when we carry out a formal evaluation where people of various age groups play the game. Based on their feedback, we will carry out a formal evaluation, approved by our University's ethics board.

This game forms part of a project being carried out at our university aimed at developing tools that can influence healthy shopping behaviour among ecommerce shoppers.

\section{RELATED WORK}

The use of games in promoting healthy eating habits is an active research area. Grimes et al. [4] developed OrderUp, a game where the player takes on the role of a server in a restaurant whose goal is to recommend the healthiest meal from a menu item as quickly as possible. The healthiness of a meal is determined by identifying the nutritional value of the meal from popular restaurants chains in the US and by searching online recipe websites. The game is targeted at African Americans living in southeastern
United States. This game differs from our game ShopRight presented in this paper. ShopRight aims to promote healthy shopping habits; we believe that because people will mostly cook what they shop for, shopping for healthy foods will likely lead them to eat healthy foods. Thus, promoting healthy eating habits at the point of shopping could likely lead to them cooking healthier foods. In addition, because families in Canada still spend about $70 \%$ of their food budget cooking at home ${ }^{6}$, it is important to influence consumers to spend this budget on healthier options.

Pollak et al. [5] developed Time to Eat, a game that aims to promote healthy eating habits. In Time to Eat, players are assigned a virtual pet that they have to care for. The players care for their pets by sending pictures of the foods they (players) eat during the day. The healthier the food, the well cared for the pet is. This game also differs from our game, ShopRight because our game proposes to influence healthy eating habits by influencing healthy shopping habits among consumers.

LunchTime [6], is a slow-casual, multi-player game that was developed to promote healthy meal choices among players while eating out. In the game, the player takes on the role of a customer in a restaurant who is presented with a menu. The player's goal is to select the meal that is best for their chosen health goal. Similar to OrderUp, LunchTIme aims to influence only consumers who eat out and not those who shop for and cook their own meals. ShopRight, on the other hand, proposes to influence healthy eating from the point of shopping of consumers.

\section{SHOPRIGHT GAME DESIGN}

ShopRight is currently an interactive single player game. The objective of the game is to shop for healthy foods while destroying the unhealthy ones. Shopping for healthy foods and destroying unhealthy ones contribute to the player's heart health. If on the other hand, a player destroys a healthy food or shops for an unhealthy one, the player's heart health reduces. In this section, we briefly describe the game's design and rules. We also introduce the architecture of the game. The game is currently a prototype at a very early stage of development. ShopRight is part of a larger project that aims to develop a platform that influences healthy shopping habits among e-commerce shoppers.

\footnotetext{
${ }^{6}$ https://www.dal.ca/news/2017/12/13/canadians-will-spendmore-in-restaurants-in-2018--canada-s-food-.html
} 


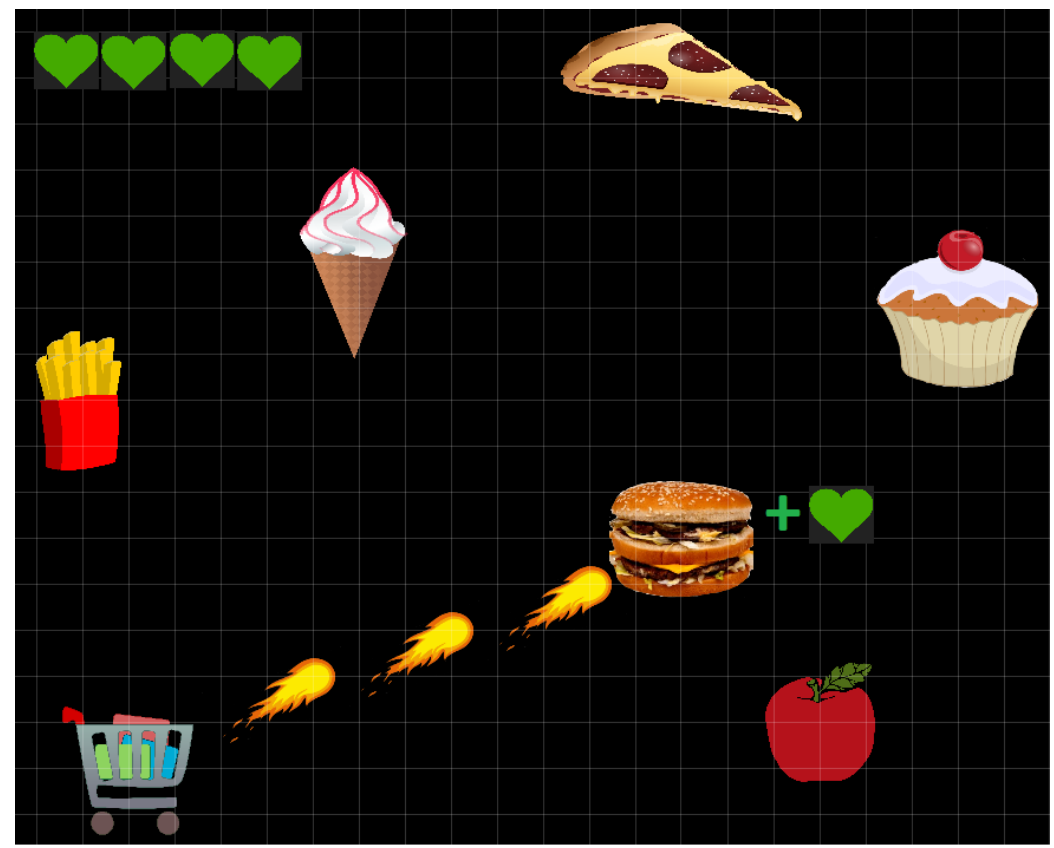

Figure 1: Screenshot of ShopRight game

\subsection{ShopRight's gameplay}

ShopRight aims to influence e-commerce shoppers to buy healthier foods. In the context of this game, healthy foods are fruits and vegetables. We hypothesize that if consumers shop for healthier foods, they will likely eat healthy foods. ShopRight is currently a web-based game that can only be played on a web browser. Players are allowed to register an account in order for their history to be saved. In the game, the player is represented by the shopping cart (as shown in figure 1). The player can control the shopping cart by using the arrow keys on the keyboard or their mouse. When they start the game, players are attacked by five unhealthy foods as shown in figure 1. The unhealthy foods quickly move towards the shopping cart (which represents the player) to consume the player. The player has to quickly move around the game while simultaneously shooting the bad foods as seen in figure 1 . Whenever the player successfully kills any of the bad foods, his/health increases. A player's health is represented by the number of green hearts they have. The more green hearts a player has, the healthier they are. When a player gets caught by an unhealthy food, the number of healthy hearts they have reduces.

To ensure that the players are able to differentiate between unhealthy and healthy foods, at random intervals, a healthy food pops up in the game such as the apple in figure 1. Although the game is a fast-paced one, the players are meant to be conscious of the different food types, thus, they should be able to identify a healthy food such as the apple and not shoot it, but rather, add the healthy food to their cart by placing the cart/moving the cart over the food. If a player is able to add a healthy food to their cart, their health (the number of green hearts in figure 1) increases. In a case where the player accidentally shoots a healthy food instead of adding it to their cart, the number of healthy hearts they have reduces by one. The game ends when a player loses all their healthy hearts.

\subsection{System architecture and implementation}

ShopRight was designed as part of a web-based project which aims to persuade e-commerce shoppers to shop for healthier foods. We thus implemented the game as a web-based one for now. We developed the game using GameMaker studio ${ }^{7}$. We chose to use GameMaker because of its built-in scripting programing language called Game Maker Language, GML. GML is easy to learn and use and does not require one to have knowledge of other complex programing languages. In addition, GML allows people to easily develop interactive games in no time.

ShopRight's game components were implemented using some of the paradigms for game design described by Duke [7]. In the following section, we briefly describe how some of the gaming elements of Duke [7] were implemented in ShopRight.

\subsubsection{Scenario}

The scenario describes the plot of the game, the starting conditions and circumstances leading to

\footnotetext{
${ }^{7}$ https://www.yoyogames.com/
} 
play [7]. The scenario of ShopRight is that of a virtual shopping experience where players earn virtual health by attacking unhealthy foods while trying not to be consumed by them and also by adding healthy foods to their shopping cart.

\subsubsection{Pulse}

According to Duke [7], a pulse is an event that is introduced into a game being played to shift the attention or focus of the player. It could be designer-induced or player-induced. In ShopRight, we adopted the designer-induced pulse option in the form of healthy foods. Healthy foods (such as the apple in figure 1) pop up from time to time. Players are meant to add such healthy foods to their shopping cart while avoiding and shooting unhealthy foods.

\subsubsection{Cycle sequence}

Cycle sequence describes a game's complete cycle from the beginning to how it ends [7]. ShopRight starts with a player being attacked by five unhealthy foods and ends when the player either shoots all unhealthy foods or when the healthy foods capture the player. The more unhealthy foods the player shoots, the more hearts they gain and vice versa.

\subsubsection{Steps to play}

The steps to play in ShopRight include shooting unhealthy foods, moving away quickly from unhealthy foods because they would consume the player, and adding healthy foods to the cart when they pop-up.

\subsubsection{Rules}

The rules of the game are straightforward and include: navigating away from unhealthy foods as quickly as possible, shooting the unhealthy foods and adding healthy foods to the shopping cart.

\subsubsection{Roles}

In ShopRight, there is only one role the players can adopt; the role of a shopper.

\subsubsection{Symbology}

Symbology describes visual aids that represent characteristics of the game [7]. In ShopRight, because the game is to encourage healthy shopping habits, we used the image of a shopping cart to represent the player. In addition, we want shoppers to visually differentiate between healthy and unhealthy foods, thus we used the image of both categories of food. Furthermore, because we wanted to associate unhealthy foods with the possibility of heart-related diseases which is a common consequence of unhealthy eating habits [1], we used the symbol of a heart to symbolize the health of the player based on their performance in the game. Finally, we used the colour green for the health of a player's heart instead of red or another colour. Red has been shown to impair performance compared to green among other reasons because it is associated with danger [8]. Green, on the other hand, has been shown to be beneficial for creative performance [9].

\section{EVALUATION}

In order to determine what shoppers think about the game, we asked a few lab mates and friends to give us their opinion of the game. Note that this was not a formal evaluation as the game is still in the design phase. The responses we got include the following:

- The background of the game should be that of a grocery store instead of the current one.

- There could be levels in the game; players could progress to the next level based on their performance at the current level.

- The game should be multi-player where players compete against each other making it more competitive.

- With the multi-player game, having a leaderboard could make the game more competitive.

- The game could use more persuasive strategies to influence people to actually use the game.

As stated earlier, ShopRight is still being refined. We plan to integrate more features based on the feedback we described above and then carry out a formal evaluation approved by the ethics board of our university.

\section{CONCLUSION AND FUTURE WORK}

Unhealthy eating habits and a sedentary lifestyle have been identified as the top two causes of obesity and weight gain. With the health problems associated with obesity and weight gain, it is imperative to put measures in place to influence people to eat healthy and maintain an active lifestyle. While a lot of research has been done on influencing people to be active, there is still room for more research in the area of promoting healthy eating habits especially by influencing people to shop for healthy food. To contribute to research in the area of shopping for healthy foods, we present the prototype of ShopRight, a game that promotes healthy shopping habits. In ShopRight, points are awarded when players "shop" for healthy foods and "destroy" the unhealthy options as quickly as possible. The aim of the game is to influence shoppers to quickly and easily identify and differentiate between healthy and unhealthy foods when shopping. In the future, we plan to incorporate the suggestions listed in section 4 and carry out a formal evaluation of the game. We also plan to incorporate harder tasks such as identifying foods with high sugar/fat content. 


\section{REFERENCES}

[1] T. Navaneelan and T. Janz, "Adjusting the scales: Obesity in the Canadian population after correcting for respondent bias,"

Statistics Canada Catalogue, Health at a Glance, vol. 82-624-X.

[2] S. Bischoff, Y. Boirie, and T. Cederholm, "Towards a multidisciplinary approach to understand and manage obesity and related diseases," Clinical Nutrition, vol. Volume 36, no. Issue 4, p. pp 917-938, 2017.

[3] R. Orji, L. E. Nacke, and C. Di Marco, "Towards Personality-driven Persuasive Health Games and Gamified Systems," in Proceedings of the $2017 \mathrm{CHI}$ Conference on Human Factors in Computing Systems CHI '17, 2017, p. pp 1015-1027.

[4] A. Grimes, V. Kantroo, and R. Grinter, "Let's play!: mobile health games for adults," in Proceedings of the 12th ACM international conference on Ubiquitous computing, 2010, pp. 241-250.
[5] J. Pollak, G. Gay, S. Byrne, and E. Wagner, "It's time to eat! Using mobile games to promote healthy eating," IEEE Journal on Pervasive Computing, vol. 9, no. 3, pp. 2127, 2010.

[6] R. Orji, J. Vassileva, and R. Mandryk, "LunchTime: a slow-casual game for longterm dietary behavior change," Personal and Ubiquitous Computing, 2013.

[7] R. D. Duke, "A Paradigm for Game Design," Simulation \& Games, vol. 11, no. 3, pp. 364-377, Sep. 1980.

[8] A. Elliot, M. Maier, A. Moller, and R. Friedman, "Color and psychological functioning: The effect of red on performance attainment.," Journal of experimental psychology: General, vol. 136, no. 1, p. 154, 2007.

[9] S. Lichtenfeld, A. Elliot, and M. Maier, "Fertile green: Green facilitates creative performance," Personality and Social Psychology, vol. 38, no. 6, pp. 784-797, 2012. 